\title{
Training and evaluation of a knowledge-based model for automated treatment planning of multiple brain metastases
}

\author{
Vishruta A. Dumane, Tsu-Chi Tseng, Ren-Dih Sheu, Yeh-Chi Lo, Vishal Gupta, Audrey Saitta, Kenneth E. \\ Rosenzweig, Sheryl Green
}

Departmentof Radiation Oncology, Icahn School of Medicine at Mount Sinai, New York, NY 10029, USA

Correspondence to: Dr. Vishruta A. Dumane, Department of Radiation Oncology, Icahn School of Medicine at Mount Sinai, 1184 5th Avenue, Box 1236, New York, NY 10029, USA. E-mail: vishruta.dumane@mountsinai.org

How to cite this article: Dumane VA, Tseng TC, Sheu RD, Lo YC, Gupta V, Saitta A, Rosenzweig KE, Green S. Training and evaluation of a knowledge-based model for automated treatment planning of multiple brain metastases. J Cancer Metastasis Treat 2019;5:42. http://dx.doi.org/10.20517/2394-4722.2019.08

Received: 14 Jan 2019 First Decision: 12 Feb 2019 Revised: 25 Mar 2019 Accepted: 15 Apr 2019 Published: 14 May 2019

Science Editor: Lucyna Kepka Copy Editor: Cai-Hong Wang Production Editor: Huan-liang Wu

\begin{abstract}
Aim: Volumetric modulated arc therapy (VMAT) has been utilized to plan and treat multiple cranial metastases using a single isocenter due to its ability to provide steep dose gradients around targets as well as low doses to critical structures. VMAT treatment is delivered in a much shorter time compared to using a single isocenter for the treatment of each lesion. However, there is a need to develop methods to reduce the treatment planning time for these cases while also standardizing the plan quality. In this work we demonstrate the use of RapidPlan, which is knowledge-based treatment (KBP) planning software to plan multiple cranial SRS cases.
\end{abstract}

Methods: The 66 patient plans with 125 lesions (range 1-4, median 1) were used to train a model. In addition, the model was validated using 10 cases that were previously treated and chosen randomly. The clinical plans were compared to plans generated by RapidPlan for target coverage and critical organ dose.

Results: Coverage to the target volume, gradient index, conformity index and minimum dose to the target showed no significant difference between the original clinical plan vs. the plan generated by KBP. A comparison of doses to the critical organs namely the brainstem, brain, chiasm, eyes, optic nerves and lenses showed no significant difference. Target dose homogeneity was slightly better with the clinical plan, however this difference was also statistically insignificant. 
Conclusion: This work demonstrates that KBP can be trained and efficiently utilized to help not only speed up the planning process but also help standardize the treatment plan quality.

Keywords: Brain metastases, radiotherapy, volumetric modulated arc therapy, knowledge-based planning, stereotactic, radiosurgery

\section{INTRODUCTION}

Volumetric modulated arc therapy (VMAT) is a radiation treatment planning and delivery technique that has been investigated and clinically applied for all disease sites including malignancies of the head and neck, thorax, abdomen and pelvis, and compared to static field intensity modulated radiation therapy (IMRT), VMAT has been shown to reduce treatment time and monitor units (MU), making it an attractive radiotherapy delivery technique ${ }^{[1]}$. Recent reports have established VMAT as a treatment technique for delivering cranial stereotactic radiosurgery $(\mathrm{SRS})^{[2-4]}$ providing optimal dose distributions while improving the efficiency of treatment delivery. Conventionally, SRS uses one isocenter per lesion and requires multiple isocenters for multiple lesions. This not only prolongs the delivery time reducing patient throughput but is also uncomfortable for the patient. The use of a single isocenter to treat multiple cranial metastases has been accomplished using VMAT producing highly conformal dose distributions while reducing treatment times. Target coverage and dose gradients produced by this technique have been shown to be comparable to Gamma Knife-based SRS ${ }^{[5,6]}$. After the initial feasibility studies, reports have been presented outlining further refinement of the procedure, dosimetric indices as well as plan quality parameters for single isocenter VMAT in radiotherapy treatment of multiple cranial metastases ${ }^{[7,8]}$. Depending on the complexity of the case however, planning for these cases can be very time consuming and reports have expressed the need to shorten the planning time, thereby aiming to improve the efficiency.

Knowledge-based planning (KBP) is a new paradigm in treatment planning and is a shift towards the direction of automating the planning process ${ }^{[9,10]}$. KBP utilizes the dose distributions of prior plans to build a model that can predict the same for new patients. KBP has been effectively utilized to generate high quality treatment plans with consistency utilizing IMRT or VMAT for malignancies at various sites including prostate, lung, liver, head and neck as well as intracranial stereotactic radiotherapy ${ }^{[1-15]}$. Although comprehensive studies describing prediction methodologies that make precise estimations of SRS plan quality metrics have been made using in-house systems ${ }^{[15]}$, clinical application of a commercial KBP system for treatment planning of multiple intracranial lesions with VMAT using a single isocenter has yet to be performed. Recently RapidPlan (TM), which is commercial KBP software from Varian Medical Systems, Palo Alto CA has become available for clinical use. In this study we train a knowledge-based model using RapidPlan for multiple cranial lesions treated at our institution with VMAT. We then retrospectively apply this model to compare the results of previously planned and treated cases.

\section{METHODS}

\section{Treatment planning}

VMAT plans were generated for 91 patients with a total of 139 lesions (range 1-4, median 1) in Eclipse V13.6 (Varian Medical Systems, Palo Alto, CA USA) from June 2017 to November 2018. Each case was planned with a single isocenter using 3 to 6 arcs. The location of the isocenter was based on the geometric center of the lesions to be treated. The arcs used were non-coplanar. Their distribution in terms of full arcs $v s$. partial arcs, couch angles, collimator angles were such that they best covered the group of lesions to be treated within that single isocenter. The arcs were chosen to avoid situations where there were two targets in the same leaf track in order to avoid excess dose to the brain. The algorithm used for optimization with VMAT was the progressive resolution optimizer and dose calculation was performed with analytical anisotropic 
algorithm using a $1 \mathrm{~mm}$ dose calculation grid size. X-ray energy used was 6 flattening filter free (FFF) and dose rate for planning was $1400 \mathrm{MU} / \mathrm{min}$. Contouring of the gross tumor volume (GTV) and the critical structures such as the brain, brainstem, chiasm, optic nerves and tracts, eyes and lenses closely followed previously published guidelines ${ }^{[7,8]}$. At our institution, no margin is used to convert GTV to planning target volume (PTV). The range of the PTV was from 0.1-7 cm3. The gradient index (GI) was defined as the ratio of volume covered by the $50 \%$ isodose line to that covered by the $100 \%$ isodose $\mathrm{e}^{[16]}$. The conformity index (CI) and the homogeneity index (HI) were chosen for plan evaluation. The CI was defined as the ratio of the volume covered by the $100 \%$ isodose to the volume covered by the PTV. The HI was taken as the maximum PTV dose to the prescription dose. Dose prescribed in a single fraction was $20 \mathrm{~Gy}, 18 \mathrm{~Gy}$ or $16 \mathrm{~Gy}$ and was decided based on the size and volume of the lesion and its proximity to critical organs. The constraints and strategy for optimization was similar to that previously published $\mathrm{d}^{[7,8,18,19]}$. The dose constraints used for planning are shown in Table 1.

\section{KBP with RapidPlan}

RapidPlan is a treatment planning application developed by Varian medicalsystems that utilizes a knowledge-based approach. Previously accepted clinical plans are taken from which data are extracted which include the volumes of the OARs and PTVs, percentage of the overlap volume for each OAR with the target, percentage of the OAR volume that is out of the field for each OAR, prescription dose, structure dose-volume histogram (DVH) and geometry based expected DVH for each OAR. The geometry based expected dose is a measure of dose received by a portion of an OAR if only the patient anatomy and desired target dose are to be considered. Principle component analysisis conducted on this extracted data and the principle components are used to build a DVH estimation model ${ }^{[17]}$. When a treatment plan is to be generated for a new patient, the RapidPlan model will create DVH estimates for the OARs based on the anatomy for that particular case. These DVH estimates will then be used as part of the objectives for optimization to achieve the dosimetric goals for that patient.

\section{Model training}

To create the RapidPlan DVH estimation model, we selected 66 patients with 125 lesions (range 1-4, median 1). The model was trained for multiple target dose levels, namely high risk, intermediate risk and a low risk PTV. These levels of risk for the PTVs were matched accordingly in both the training and validation. For all single lesion cases, the plan had only 1 target, which was matched to high dose level. If the plan had 2 targets that were prescribed to the same dose level, then they both were matched to the highest dose level. However, if they went to different dose levels, the target receiving higher prescription was matched to the higher dose level and the one receiving the next dose level was matched to the intermediate dose. Similarly if a plan had 3 targets, each prescribed to different dose levels, the targets were matched correspondingly, i.e., high to high, intermediate to intermediate and low to low. However if they went to 2 different dose levels, the target(s) with the highest prescription dose would be matched to high and the target(s) with the next dose level would be matched to intermediate dose level. Similarly if all the 3 went to the same dose level, all of them would be matched to high dose. This methodology of matching is recommended by the training software. The OARs included for training were brain, brainstem, chiasm, optic nerves, eyes and lenses. The training process consisted of identifying the geometric outliers and the dosimetric outliers. The geometric outliers are typically cases were the PTV and/or OAR volumes, shapes and overlaps differ substantially from the majority of the training set. Dosimetric outliers are cases where the clinical DVH differs substantially from the estimated DVH. Geometric outliers can be identified using regression plots, which illustrate the correlation between the best prediction of the DVH and the most likely geometric parameter that would be responsible for that DVH such as the volume of the structure or the overlap with the target or a combination of both. Geometric outliers are points that are typically identified as points that fall far away from the regression line or that are substantially isolated from it. Similarly, dosimetric outliers can be identified using something called residual plots that correlate the best estimation of the DVH to the actual clinical DVH for 
Table 1. Table summarizing dosimetric criteria

\begin{tabular}{lll}
\hline Structure & Parameter & Objective \\
\hline PTV & $D_{\max }(\%)$ & $\leq 125 \%^{\star}$ \\
& $D_{\min }(\%)$ & $\geq 90 \%^{\star}$ \\
& $V_{100}(\%)$ & $\geq 98 \%^{\star}$ \\
Brainstem & $D_{\max }(\mathrm{Gy})$ & $\leq 13 \mathrm{~Gy}^{\star}$ \\
Brain & $V_{7 G y}(\%)$ & $\leq 5 \% \%^{\star}$ \\
& $V_{12 G y}\left(\mathrm{~cm}^{3}\right)$ & $\leq 8 \mathrm{~cm}^{3 \star}$ \\
Optics & $V_{10 G y}\left(\mathrm{~cm}^{3}\right)$ & $\leq 10 \mathrm{~cm}^{3 \star}$ \\
Lens & $D_{\max }(\mathrm{Gy})$ & $\leq 8 \mathrm{~Gy}^{\star}$ \\
\hline
\end{tabular}

${ }^{*}$ Signifies used as limiting constraints, while the others are used as guidelines. $D_{\max }(\%)$ is the maximum dose to the structure in \%, $D_{\min }(\%)$ is the minimum dose to the structure in $\%, V_{100}(\%)$ is the volume of the structure in $\%$ that receives $100 \%$ of the prescription dose. $D_{\max }(\mathrm{Gy})$ is the maximum dose to the structure in $\mathrm{Gy}$ and $\mathrm{V}_{\mathrm{XG}}(\%)$ is the \% volume of the structure receiving $X \mathrm{~Gy}$, and $\mathrm{V}_{\mathrm{YGy}}\left(\mathrm{cm}^{3}\right)$ is the absolute volume of the structure receiving $Y$ Gy. At our institution, the maximum dose constraint to the brainstem is used only if target overlaps with the brainstem. PTV: planning target volume

the training case. A case is considered to be a dosimetric outlier when the clinical DVH differs substantially from the DVH estimated by the model. By removing the geometric and dosimetric outliers, and re-training the model, the ability of the model to estimate the DVHs is measured. A minimum of 20 patients/cases are required to successfully train the model.

\section{Optimization objectives}

Once the model has been trained and reviewed, optimization objectives are added to the model. It is critical to mention at this point that these estimation models can predict achievable OAR DVHs for individual patient anatomy; however optimization objectives are required to achieve these dosimetric goals. These dose-volume objectives and the priorities are estimated by the model for the selected structure and are automatically loaded when the model is selected to create a plan for a new patient.

\section{Model validation}

Ten patients were chosen for validation. Among the 10 patients, 6 had 1 lesion each, 2 had 2 lesions each, 1 had 3 lesions and 1 had 4 lesions. The validation process consists of comparing the clinical DVH to that obtained from the plan using the model and then evaluating the plan for clinical acceptability. The dosimetric parameters extracted with respect to target coverage were maximum dose to the PTV, minimum dose to the PTV, volume of the PTV covered by $100 \%$ of the prescription dose, i.e., PTV V100, GI, CI and HI, all of which have been defined earlier. With respect to the critical organs, the dosimetric parameters noted were the maximum dose to the brainstem, brain, chiasm, optic nerve, eyes and lenses. The V7 Gy, V12 Gy and V10 Gy to the brain were also noted. Significance testing between the dosimetric results of the clinical plan $v s$. the plan generated by the model, i.e., the validation plan was performed by using the Wilcoxon sign rank test, which is a non-parametric test for matched pairs of data.

\section{RESULTS}

Although 91 patients with a total of 139 lesions were planned, we could only use 66 of them with a total of 125 lesions due to limitations in Eclipse V 13.6 as some of the target volumes were too small to be extracted. Comparison of dosimetric parameters for target coverage between the original clinical plan and the corresponding knowledge-based validation plan using RapidPlan are shown in Table 2. Similarly in Table 3, is the comparison of the dosimetric parameters for OAR sparing between the original clinical plan and the validation plan. In Table 4, for the validation cases, the diameter and volume of each lesion treated in a single radiotherapy session is indicated. For multiple lesion cases, i.e., 2.3 and 4 lesions, the total volume of all lesions treated as well as the maximum diameter is indicated. In Table 5, is shown the distribution of the lesions to the various risk levels. Each validation plan was generated within 30 minutes without human 
Table 2. Comparison of dosimetric parameters for target coverage between the original clinical plan and the corresponding knowledge based plan, i.e., Rapidplan. Wilcoxon sign-rank test shows that the dosimetric results with respect to target coverage show no statistically significant difference between the two planning techniques

\begin{tabular}{llllc}
\hline Structure & \multicolumn{1}{c}{ Parameter } & Clinical plan & KBP & P value \\
\hline PTV & $D_{\max }(\%)=H I$ & $118 \% \pm 3.4 \%$ & $120 \% \pm 2.6 \%$ & 0.05 \\
& $D_{\min }(\%)$ & $97 \% \pm 2.8 \%$ & $97 \% \pm 3.3 \%$ & 0.86 \\
& $V_{100}(\%)$ & $100 \% \pm 0.8 \%$ & $100 \% \pm 1.1 \%$ & 1.00 \\
& I & $1.5 \pm 0.2$ & $1.5 \pm 0.2$ & 1.00 \\
& GI & $4 \pm 0.6$ & $4.3 \pm 0.7$ & $<0.01$ \\
\hline
\end{tabular}

$\mathrm{Cl}$ : conformity index; GI: gradient index; HI: homogeneity index; KBP: knowledge based plan; PTV: planning target volume

Table 3. Comparison of dosimetric parameters for critical organs between the original clinical plan and the corresponding knowledge based plan, i.e., Rapidplan. Wilcoxon sign-rank test shows that the dosimetric results with respect to target coverage show no statistically significant difference between the two planning techniques

\begin{tabular}{lllll}
\hline Structure & Parameter & Clinical plan & KBP & P value \\
\hline Brainstem & $D_{\max }(\mathrm{Gy})$ & $2.2 \pm 3.7$ & $2.2 \pm 3.5$ & 1.00 \\
Brain & $D_{\max }(\mathrm{Gy})$ & $20.8 \pm 1$ & $21.2 \pm 1$ & 0.02 \\
& $V_{7 G y}(\%)$ & $0.7 \% \pm 0.8 \%$ & $0.7 \% \pm 0.8 \%$ & 1.00 \\
& $V_{12 G y}\left(\mathrm{~cm}^{3}\right)$ & $3.5 \pm 2.8$ & $4 \pm 3.4$ & 0.13 \\
& $V_{10 G y}\left(\mathrm{~cm}^{3}\right)$ & $5.2 \pm 3.9$ & $5.9 \pm 4.9$ & 0.16 \\
Chiasm & Mean $(\mathrm{Gy})$ & $0.6 \pm 0.3$ & $0.7 \pm 0.4$ & 0.06 \\
Eye & $D_{\max }(\mathrm{Gy})$ & $0.5 \pm 0.5$ & $0.5 \pm 0.5$ & 0.63 \\
Optic nerve & $D_{\max }(\mathrm{Gy})$ & $0.3 \pm 0.5$ & $0.4 \pm 0.5$ & 0.30 \\
Lens & $D_{\max }(\mathrm{Gy})$ & $0.3 \pm 0.4$ & $0.3 \pm 0.4$ & 0.38 \\
\hline
\end{tabular}

Cl: conformity index; GI: gradient index; KBP: knowledge based plan

Table 4. Table showing the diameter and volume of each lesion treated in a single radiotherapy session for each of the validation cases. For multiple lesion case i.e., 2,3 and 4 lesions, the total volume of all lesions treated as well as the maximum diameter is indicated

\begin{tabular}{lccc}
\hline Case & No. of lesions & Diameter $\mathbf{( c m )}$ & Volume $\left(\mathbf{c m}^{\mathbf{3}}\right)$ \\
\hline Case 1 & 1 & 1 & 3.9 \\
Case 2 & 1 & 0.5 & 0.4 \\
Case 3 & 1 & 0.4 & 0.2 \\
Case 4 & 1 & 0.4 & 0.3 \\
Case 5 & 1 & 0.9 & 2.7 \\
Case 6 & 1 & 0.9 & 3.2 \\
Case 7 & 2 & 0.5 & 0.5 \\
Case 8 & 2 & 0.4 & 0.3 \\
Case 9 & 3 & 0.7 & 1.7 \\
Case 10 & 4 & 0.6 & 0.7 \\
\hline
\end{tabular}

Table 5. Table showing the distribution of lesions to the various risk levels

\begin{tabular}{lccc}
\hline Case & No. of lesions & Prescription (Gy) & Dose level assignment \\
\hline Case 1 & 1 & $20 \mathrm{~Gy}$ & High dose level \\
Case 2 & 1 & $18 \mathrm{~Gy}$ & High dose level \\
Case 3 & 1 & $18 \mathrm{~Gy}$ High dose level \\
Case 4 & 1 & $18 \mathrm{~Gy}$ High dose level \\
Case 5 & 1 & $18 \mathrm{~Gy}$ & High dose level \\
Case 6 & 1 & $18 \mathrm{~Gy}$ & High dose level \\
Case 7 & 2 & All 18 Gy & High dose level \\
Case 8 & 2 & All 18 Gy & High dose level \\
Case 9 & 3 & All 18 Gy & High dose level \\
Case 10 & All 18 Gy & High dose level \\
\hline
\end{tabular}

intervention. A comparison of the dose distributions between the clinical plan and the KBP plan for a single lesion, 2 lesion and a 4 lesion case are shown in Figures 1-3 respectively. A comparison of the DVHs for the 


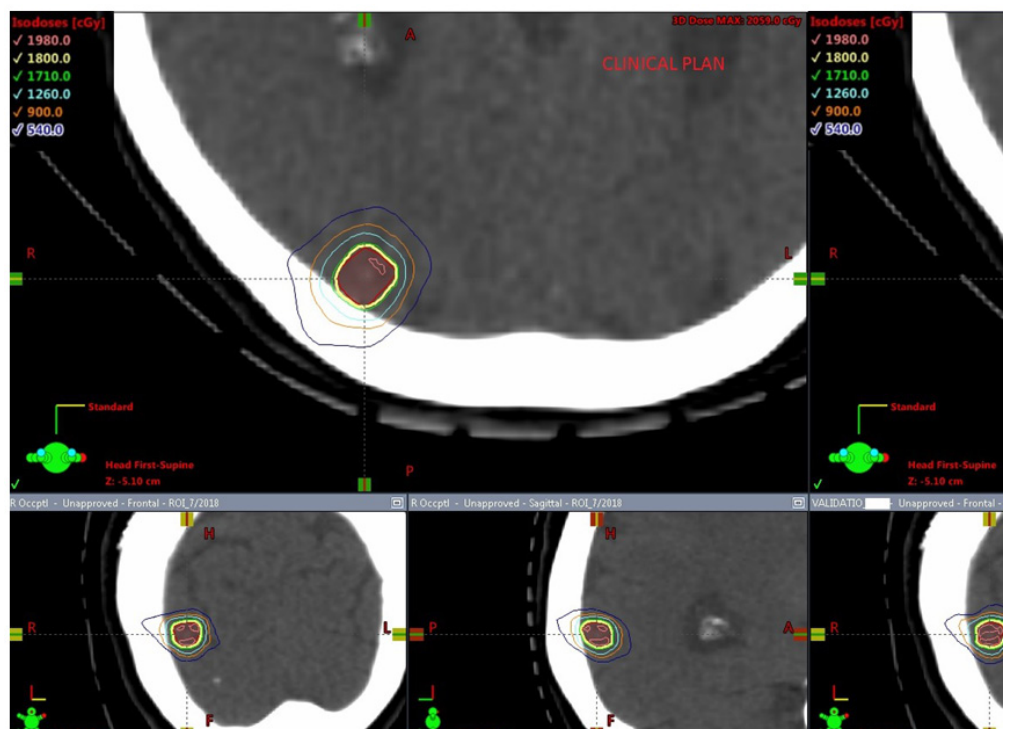

Figure 1. Comparison of dose distribution in the axial, coronal and sagittal views for a clinical plan vs. a RapidPlan for a single lesion case

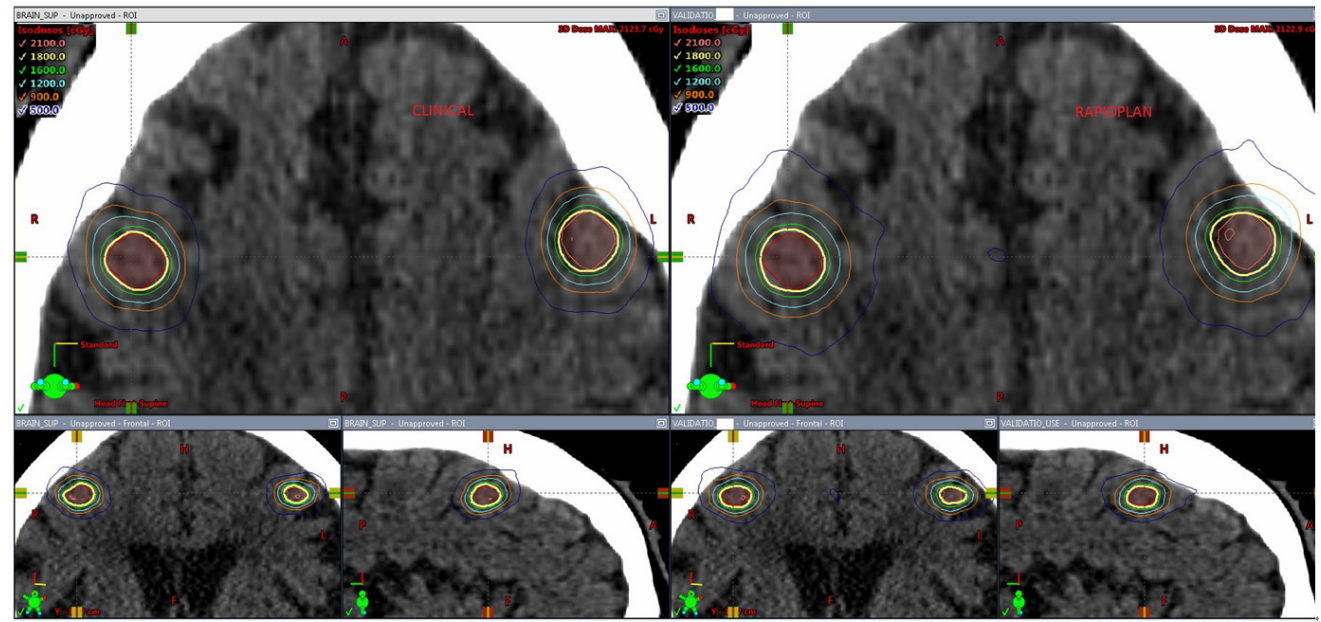

Figure 2. Comparison of dose distribution in the axial, coronal and sagittal views for a clinical plan vs. a RapidPlan for a 2 lesion case

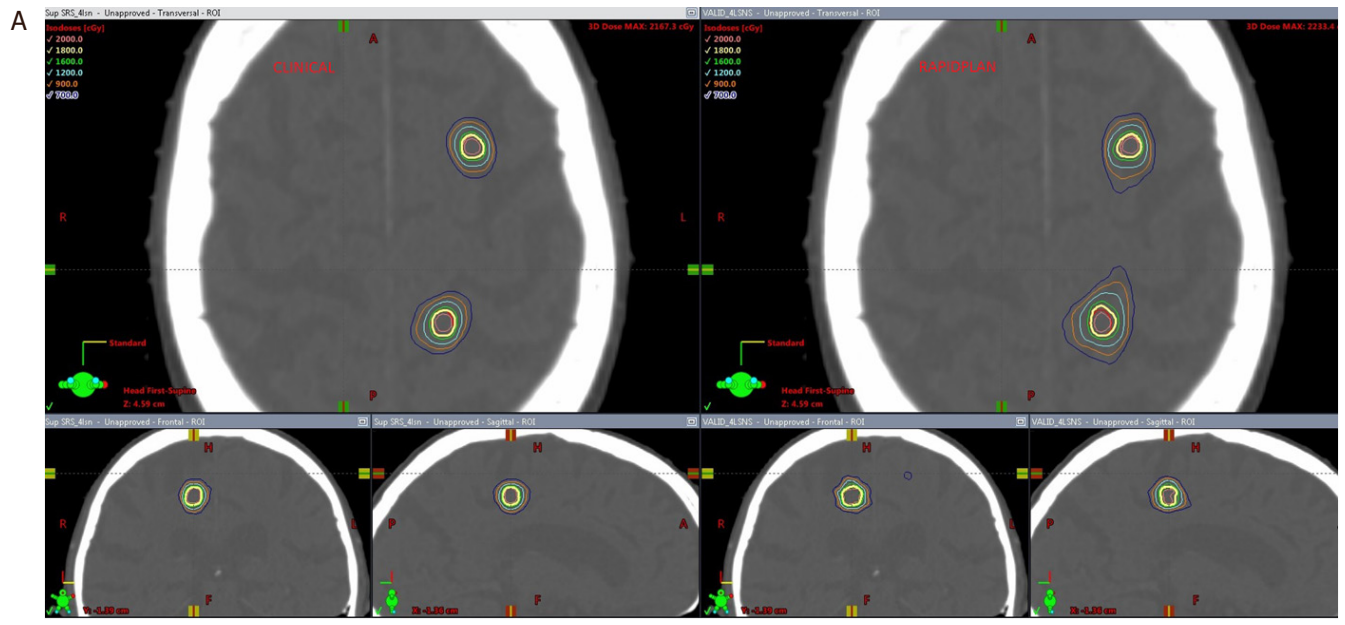




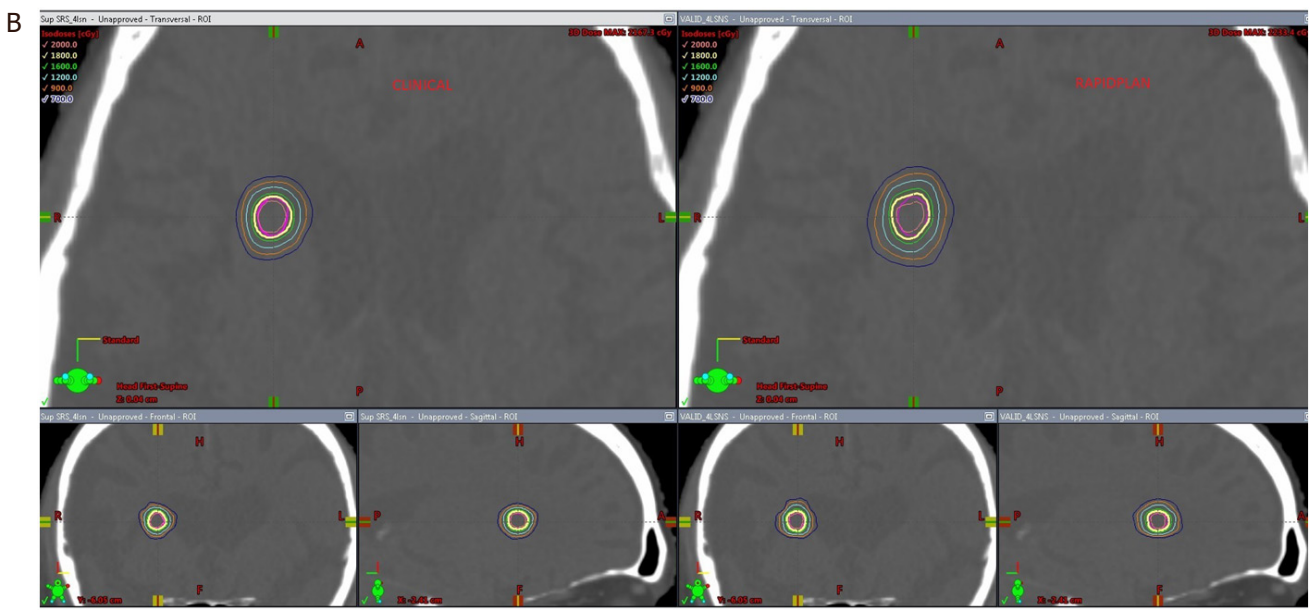

Figure 3. A: Comparison of dose distribution in the axial, coronal and sagittal views for a clinical plan vs. a RapidPlan for a 4 lesion case (first 3 lesions seen in this figure); B: comparison of dose distribution in the axial, coronal and sagittal views for a clinical plan vs. a RapidPlan for a 4 lesion case (4th lesion seen in this figure)

PTV and the critical organs for the clinical plan $v$ s. the RapidPlan for one of the single lesion cases is shown in Figure 4.

\section{PTV coverage}

The PTV coverage in terms of PTV V100, the minimum dose to the PTV showed no statistically significant difference between the clinical plan vs. the RapidPlan. Both the clinical and the RapidPlan showed no statistically significant difference in the CI. The GI was slightly higher with the RapidPlan compared to the clinical plan. The maximum dose to the PTV was higher by $2 \%$ using KBP. However these hotspots were retained within the target volume.

\section{OAR sparing}

Dose constraints for the brainstem, brain, chiasm, optic nerves, eyes and lenses were all achieved as per Table 1 for both the clinical plan as well as the KBP. No statistically significant differences were seen in the dosimetric parameters to the majority of these critical structures.

\section{Monitor units (MU) and calculated treatment time}

The total MU for the original clinical plan on average were 5,215 \pm 924, while with RapidPlan were $5503 \pm 1208$. This difference was not found to be statistically significant $(P=0.5)$.

\section{DISCUSSION}

Studies have shown that LINAC based radiosurgery plans using VMAT can produce target coverage and dose fall-off in the high dose area similar to Gamma-Knife plans ${ }^{[5,6]}$. Single isocenter cranial VMAT radiosurgery technique can produce with the major advantage being improvement in clinical efficiency. The use of FFF beams with a high dose rate of delivery at $1400 \mathrm{MU} / \mathrm{min}$ has further contributed towards to this goal. As the single isocenter VMAT technique replaces the use of multiple isocenter techniques for multiple targets, there is also a need to improve the efficiency of clinical treatment planning for these cases. Although the concept of knowledge-based planning with in-house systems has been applied to predict plan quality metrics in intracranial SRS ${ }^{[12]}$, our study has demonstrated the use of RapidPlan, which is a commercial system for automated planning of intracranial SRS. Our results indicate that both the clinical as well as the validation plan (RapidPlan) showed no significant difference with respect to target coverage, conformity index, gradient index, homogeneity as well as critical organ sparing. 

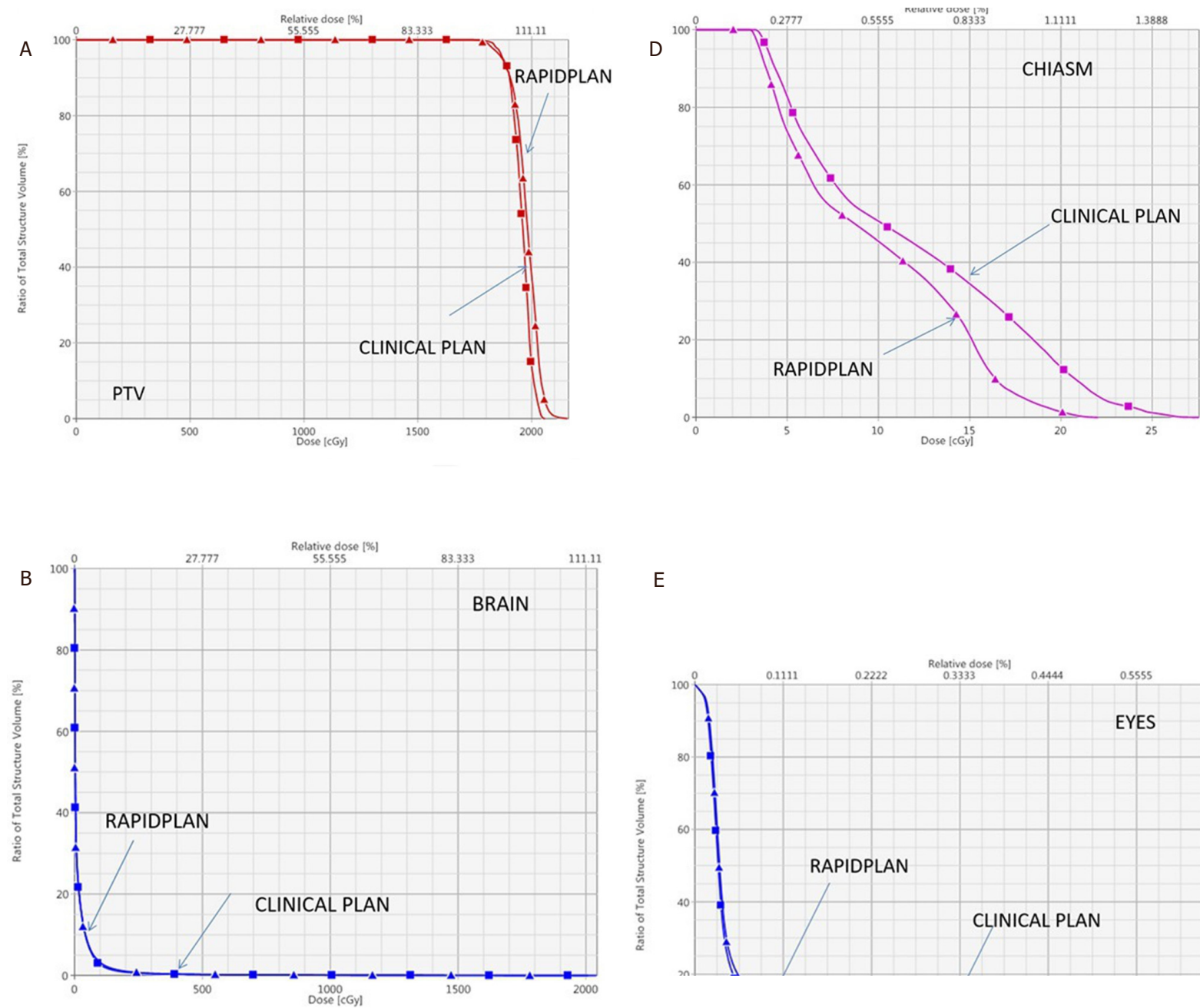

$\mathrm{E}$
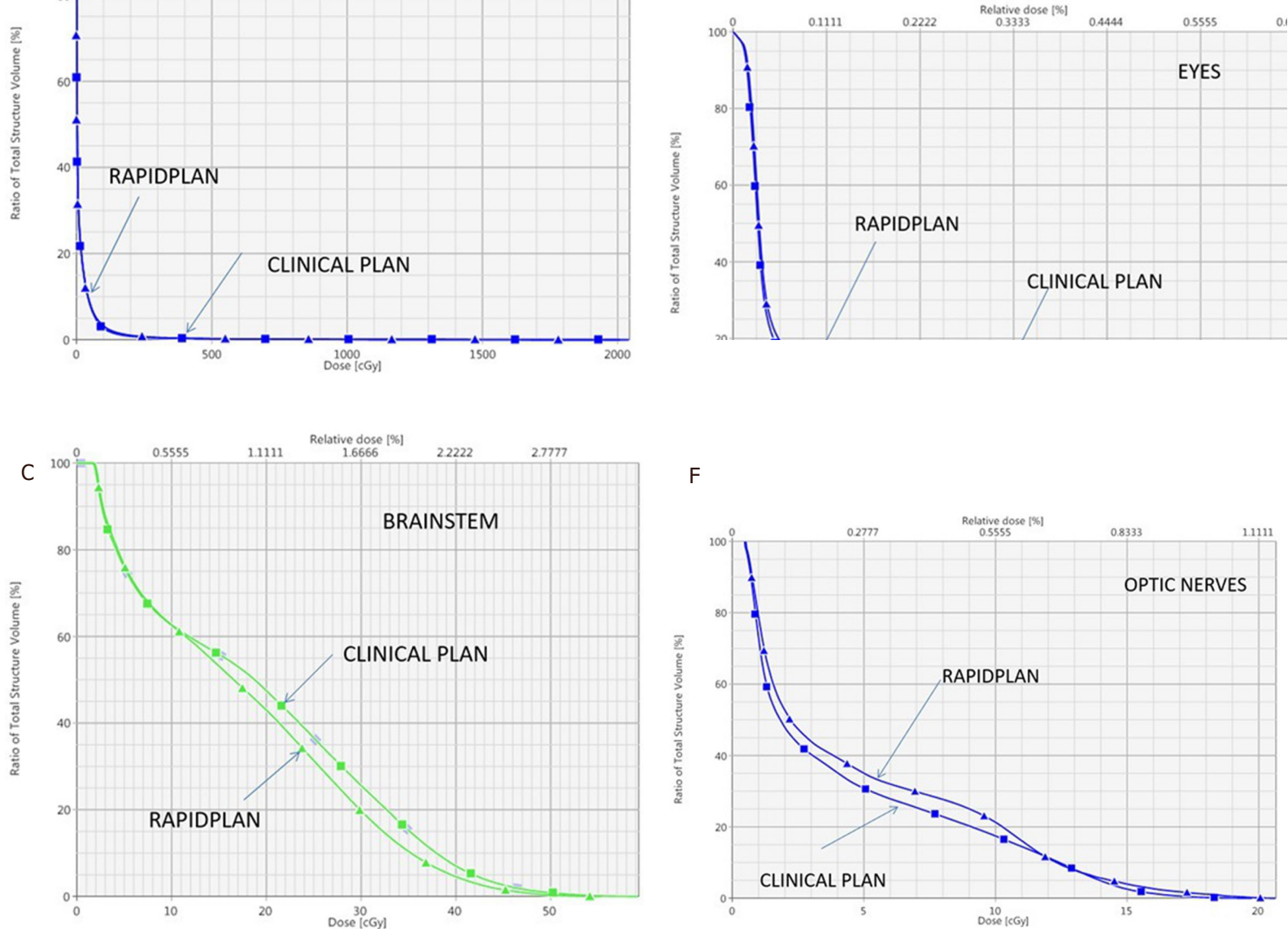

$\mathrm{F}$

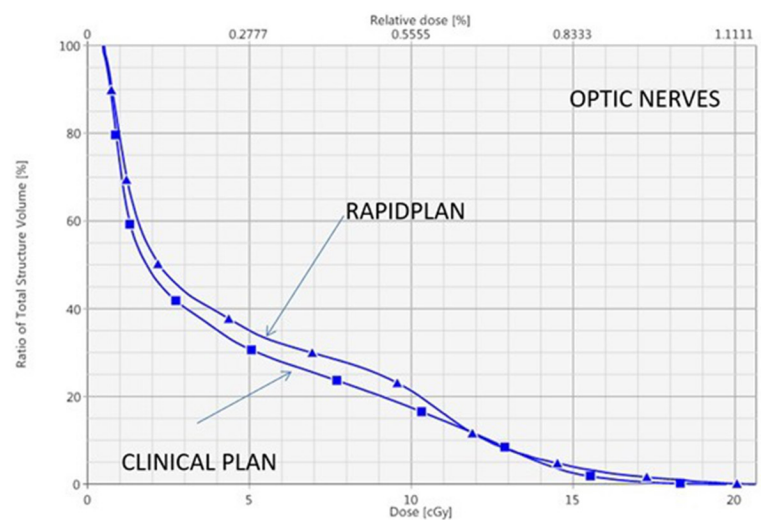

Figure 4. A: DVH comparison of target coverage for clinical plan vs. RapidPlan for the single lesion case shown in Figure 1; B: DVH comparison for the brain between the clinical plan vs. RapidPlan for the single lesion case of Figure 1; C: DVH comparison for the brainstem between the clinical plan vs. RapidPlan for the single lesion case of Figure 1; D: DVH comparison for the chiasm between the clinical plan vs. RapidPlan for the single lesion case of Figure 1; E: DVH comparison for the eyes between the clinical plan vs. RapidPlan for the single lesion case of Figure 1; F: DVH comparison for the optic nerves between the clinical plan vs. RapidPlan for the single lesion case of Figure 1. DVH: dose-volume histogram 
All the cases for training and validation were chosen without any pre-selection criteria. The limitation of this study is that it was trained and tested only with a maximum of 4 lesions per case. This was because in the cohort of patients that were treated, there were only 3 cases that had $>4$ lesions, which essentially is not enough to train a model. As we treat and acquire data on more patients that have $>4$ lesions, these can be incorporated into the model. Another limitation of this study is that the cases used for training were of smaller volume and were typically peripheral and far away from critical organs as well as from each other. This model therefore cannot be used for lesions that are overlapping with critical organs or for lesions in close proximity of each other.

The plans were slightly more inhomogeneous compared to the original clinical plans. This could be due to the fact that we used a line objective for the DVH in addition to the maximum point dose objective for the critical organ. Although the DVH for the PTV shows a longer tail, the dose inhomogeneity is contained within the target. We anticipate that with more training cases, the dosimetric results will improve. The GI was also slightly higher with the KBP plans than the recommended value of $<4$. However the plans were dosimetrically very similar. Moreover, when a typical clinical plan would take at least 2 to $3 \mathrm{~h}$ to complete, the RapidPlan gave a clinically acceptable result in under $30 \mathrm{~min}$.

The quality of the treatment plan generated by RapidPlan can only be as good as that of the treatment plans used to generate the DVH estimation model. Other technologies such as Hyperarc ${ }^{\bullet}$ are available from Varian Medical Systems that can automate SRS planning, however, they are currently unavailable with RapidPlan. This study demonstrates the feasibility of using RapidPlan as it pertains to a limited number of lesions $(\leq 4)$. Going forward, we plan on expanding its training and application to $>4$ lesions. In the future we plan to expand this to $>4$ lesions and perform a comparison with plans generated using Hyperarc.

In conclusion, we have developed an efficient method for treatment planning of multiple cranial SRS lesions using VMAT and a single isocenter. This is a step not only towards a reducing the treatment planning time but also providing the planner a guide on the achievable dose distribution for the given case, thereby helping to standardize the quality of the treatment plans.

\section{DECLARATIONS}

\section{Authors' contributions}

Built the model, studied design, performed the dosimetric and statistical analysis: Dumane VA

Planned the clinical cases that were used for training and testing while building the model: Tseng TC

Designed the database used for obtaining patient treated with SRS using VMAT on Eclipse planning system: Sheu RD

Provided input towards study design and performed statistical analysis: Lo YC

Contributed towards writing the manuscript: Dumane VA, Tseng TC, Sheu RD, Lo YC, Gupta V, Saitta A, Rosenzweig KE, Green S

Participated in the study design, contouring of all the target volumes and critical organs, reviewing plans: Green $S$

\section{Availability of data and materials}

The datasets used and/or analyzed during the current study are available from the corresponding author on reasonable request.

\section{Financial support and sponsorship}

None. 


\section{Conflicts of interest}

All authors declared that there are no conflicts of interest.

\section{Ethical approval and consent to participate}

Not applicable.

\section{Consent for publication}

Not applicable.

\section{Copyright}

(c) The Author(s) 2019.

\section{REFERENCES}

1. Teoh M, Clark CH, Wood K, Whitaker S, Nisbet A. Volumetric modulated arc therapy: a review of current literature and clinical use in practice. Br J Rad 2011;84:967-96.

2. Mayo CS, Ding L, Addesa A, Kadish S, Fitzgerald TJ, Moser R. Initial experience with volumetric IMRT (RapidArc) for intracranial stereotactic radiosurgery. Int J Radiat Oncol Biol Phys 2010;78:1457-66.

3. Wolff HA, Wagner DM, Christiansen H, Hess CF, Vorwerk H. Single fraction radiosurgery using RapidArc for treatment of intracranial targets. Radiat Oncol 2010;5:77.

4. Clark GM, Popple RA, Young PE, Fiveash JB. Feasibility of single-isocenter volumetric modulated arc radiosurgery for treatment of multiple brain metastases. Int J Radiat Oncol Biol Phys 2010;76:296-302.

5. Liu H, Andrews DW, Evans JJ, Werner-Wasik M, Yu Y, et al. Plan quality and treatment efficiency for radiosurgery to multiple brain metastases: non-coplanar rapidarc vs Gamma Knife. Front Oncol 2016;6:26.

6. Thomas EM, Popple RA, Wu X, Clark GM, Markert JM, et al. Comparison of plan quality and delivery time between volumetric modulated arc therapy (RapidArc) and Gamma Knife radiosurgery for multiple cranial metastases. Neurosurgery 2014;75:409-17.

7. Clark GM, Popple RA, Prendergast BM, Spencer SA, Thomas EM, et al. Plan quality and treatment planning technique for single isocenter cranial radiosurgery with volumetric modulated arc therapy. Pract Radiat Oncol 2012;2:306-13.

8. Ballangrud Å, Kuo LC, Happersett L, Lim SB, Beal K, et al. Institutional experience with SRS VMAT planning for multiple cranial metastases. J Appl Clin Med Phys 2018;19:176-83.

9. Moore KL, Brame RS, Low DA, Mutic S. Experience-based quality control of clinical intensity-modulated radiotherapy planning. Int J Radiat Oncol Biol Phys 2011;81:545-51.

10. Appenzoller L, Michalski J, Thorstatd W, Mutic S, Moore KL. Predicting dose-volume histograms for organs-at-risk in IMRT planning. Med Phys 2012;39:7446-61.

11. Chanyavanich V, Das SK, Lee WR, Lo JY. Knowledge-based IMRT treatment planning for prostate cancer. Med Phys 2011;38:2515-22.

12. Wu B, Pang D, Simari P, Taylor R, Sanguineti G, McNutt T. Using overlap volume histogram and IMRT plan data to guide and automate VMAT planning: A head-and-neck case study. Med Phys 2013;40:021714.

13. Fogliata A, Belosi F, Clivio A, Navarria P, Nicolini G, et al. On the pre-clinical validation of a commercial model-based optimization engine: application to volumetric modulated arc therapy for patients with lung or prostate cancer. Radiother Oncol 2014;113:385-91.

14. Fogliata A, Wang PM, Belosi F, Clivio A, Nicolini G, et al. Assessment of a model based optimization engine for volumetric modulated arc therapy for patients with advanced hepatocellular cancer. Radiother Oncol 2014;9:236-48.

15. Shiraishi S, Tan J, Olsen LA, Moore KL. Knowledge-based prediction of plan quality metrics in intracranial stereotactic radiosurgery. Med Phys 2015;42:908-17.

16. Paddick I, Lippitz B. A simple dose gradient measurement tool to complement the conformity index. J Neurosurg 2006;105:194-201.

17. Yuan L, Ge Y, Lee WR, Yin FF, Kirkpatrick JP, et al. Quantitative analysis of the factors which affect the interpatient organ-at-risk dose sparing variation in IMRT plans. Med Phys 2012;39:6868-78.

18. Minniti G, Clarke E, Lanzetta G, Osti MF, Trasimeni G, et al. Stereotactic radiosurgery for brain metastases: analysis of outcome and risk of brain radionecrosis. Radiat Oncol 2011;6:48.

19. Blonigen BJ, Steinmetz RD, Levin L, Lamba MA, Warnick RE, et al. Irradiated volume as a predictor of brain radionecrosis after linear accelerator stereotactic radiosurgery. Int J Radiat Oncol Biol Phys 2010;77:996-1001. 